\title{
Low-Cycle Fatigue and Creep-Fatigue Behaviors of a Second-Generation Nickel-Based Single-Crystal Superalloy at $760{ }^{\circ} \mathrm{C}$
}

\author{
Jian Zhang ${ }^{1} \cdot$ Yuan-Yuan Guo $^{1} \cdot$ Mai Zhang ${ }^{1,2} \cdot$ Zhen-Yu Yang $^{1} \cdot$ Yu-Shi Luo ${ }^{1}$ \\ Received: 26 December 2019 / Revised: 15 February 2020 / Published online: 18 May 2020 \\ (c) The Chinese Society for Metals (CSM) and Springer-Verlag GmbH Germany, part of Springer Nature 2020
}

\begin{abstract}
Low-cycle fatigue (LCF) behaviors of a second-generation nickel-based single-crystal superalloys with [001] orientation at $760{ }^{\circ} \mathrm{C}$ have been investigated. Different strain amplitudes were introduced to investigate the creep-fatigue effects. The LCF life of none tensile holding (NTH) was higher than that of the 60-s tensile hold (TH) at any strain amplitude. As the strain amplitude was $0.7 \%$, the stacking and cross-slip dislocations appeared together at the $\gamma / \gamma$ ' coherent microstructure in both $\mathrm{TH}$ and NTH specimens. At the strain amplitude of $0.9 \%$, plenty of the cross-slip dislocations appeared in $\gamma$ channel and other dislocations were stacking at $\gamma / \gamma$ ' interfaces. However, the SFs still appeared in $\gamma$ ' phase with 60-s TH which caused cyclic softening. As the strain amplitude increased up to $1.2 \%$, the dislocations are piling up at the $\gamma / \gamma^{\prime}$ interfaces and cutting through the $\gamma$ ' phase in both TH and NTH tests, which caused cyclic hardening. The influences of strain amplitude and holding time were complicated. Different stress response behaviors occurred in different loading conditions. The surface characteristic and fracture mechanism were observed by scanning electron microscopy. This result is helpful for building the relationship of various blade fatigue failure modes, cyclic stress response and microstructure deformation under different strain amplitudes.
\end{abstract}

Keywords Low-cycle fatigue $\cdot$ Cyclic stress response behavior $\cdot$ Dislocation morphologies

\section{Introduction}

With excellent creep resistance, persistent strength and thermal fatigue resistance are at the elevated temperatures. Nickel-based single-crystal (SC) superalloys have been used as turbine blade materials in the gas turbines [1-3]. Turbine blades are usually subjected to cycle thermal stress and alternate loading, which are caused by starting up and shutdown of gas turbines. In this case, the low-cycle fatigue (LCF) failure is one of the common failure modes [4-7], and it is important to understand the LCF mechanism for development and application of the nickel-based SC superalloys at working temperatures [8].

Available online at http://link.springer.com/journal/40195.

Jian Zhang

15884418796@163.com

1 Beijing Institute of Aeronautical Materials, Beijing 100095, China

2 School of Materials Science and Engineering, University of Science and Technology Beijing, Beijing 100083, China
Fatigue life and fatigue deformation mechanisms of the nickel-based SC superalloys have been confirmed to be governed by the chemical composition, the size and volume fraction of $\gamma$ ' phase and loading conditions [9-17]. The distinct cyclic stress responses and cracking morphology of the nickel-based SC superalloys depend on slip characteristics under various loading conditions such as strain amplitude, temperatures, oxidation and hold time [18-21]. Under room temperature, the deformation mechanism was planar slip which leads to crystallographic cracking [22]. Wavy slip was recognized to be the dominate deformation mechanism under high temperature. At high temperature, coarsened and joint $\gamma$ ' phase which was influenced by loading conditions decreases the resistance of dislocation motion in the LCF deformation processes [16]. The fatigue crack propagation rate of a CMSX-2 nickel-based SC superalloys at $950{ }^{\circ} \mathrm{C}$ was slowed down through oxide-induced closure and crack-tip blunting mechanisms at low stress field intensity range $\Delta K$ [23]. Under the load-holding condition during fatigue tests, the areal crack density and mean crack length were increased than those in the non-hold tests [24]. 
The strain amplitude is a key factor that dominates the LCF life and fatigue mechanisms of nickel-based SC superalloys blade components subjecting to the engine start and cooldown at the moderate temperatures. It was confirmed that with the strain amplitude increasing, the density of dislocation increases, the plastic deformation state and microstructure switch from fully anisotropic to isotropic [15]. The cyclic stress response, including the cyclic hardening and cyclic softening, is related to dislocation or SF which depends on strain amplitude and fatigue testing temperature [25]. Li found that the slip bands can be observed and the movement of dislocation was not homogeneous at $750{ }^{\circ} \mathrm{C}$. However, the understanding of effect of different strain amplitudes from 0.7 to $1.2 \%$ on LCF behaviors of the nickelbased SC superalloys has been still far from systematization.

The actual service temperature at the extension roots and serration of the disk/blade components is approximate $760{ }^{\circ} \mathrm{C}$, and this temperature is an extremely complex stage for the deformation mode, corresponding to a transition of the deformation mechanism from planar slip at room temperature to the wavy slip at temperatures higher than $900{ }^{\circ} \mathrm{C}$ [22]. In this article, the nickel-based SC superalloy included 3\% Re and 8\% Co. Re, the most important element of the second-generation nickel-based SC superalloys, can reduce the fault energy value and increase the interface mismatch which plays an important role in the LCF life and fatigue deformation mechanisms of nickel-based SC superalloys [9]. It was found that the additions of Re and Co decrease the stacking fault (SF) energy of pure Ni from 200 to $20-30 \mathrm{~mJ} \mathrm{~m}^{2}$ [26]. And the SFs significantly affect mechanical behaviors. However, during the LCF at $760{ }^{\circ} \mathrm{C}$, the SFs just appeared in the $\gamma$ phase, whereas few was present in the $\gamma^{\prime}$ phase [27].

Therefore, it is important to understand evolution of the SFs in the $\gamma / \gamma^{\prime}$ coherent microstructure and how SFs affect the LCF behavior, which would be beneficial to the alloy design, fatigue life predication and applications. The detail of the LCF performance and damage mechanism of a second nickel-based SC superalloy as a function of the strain amplitude at $760{ }^{\circ} \mathrm{C}$ was investigated in this article.

\section{Experimental}

The chemical composition of the second nickel-based SC superalloy is listed in Table 1. The SC bars with the dimension of $15 \mathrm{~mm}$ in diameter and $150 \mathrm{~mm}$ in length and the orientation of [001] were prepared in an HRS directional solidification furnace through grain selector at a withdrawing rate of $3 \mathrm{~mm} / \mathrm{min}$. The orientation deviation of the SC bars was within $8^{\circ}$, which were determined by the X-ray back scattering Laue method. SC bars were solution-treated at $1315^{\circ} \mathrm{C}$ for $6 \mathrm{~h}$ in a vacuum, followed by furnace cooling to room temperature. Finally, they were aged at $1130^{\circ} \mathrm{C}$ for $4 \mathrm{~h}$ plus $870^{\circ} \mathrm{C}$ for $24 \mathrm{~h}$.

SC bars were machined and polished for the fatigue specimens, $90 \mathrm{~mm}$ in length and $6 \mathrm{~mm}$ in diameter of gauge length. The loading direction of specimens was parallel to the [001] crystal orientation. Fatigue tests were performed using an electro-hydraulic servo-testing machine in air at $760{ }^{\circ} \mathrm{C}$ with a variation of $\pm 2{ }^{\circ} \mathrm{C}$. The wavy form of the fatigue loading was trapezoidal, and the loading rate was $5 \times 10^{-3} \mathrm{~s}^{-1}$. In the fatigue test, the total strain amplitudes $(\Delta \varepsilon / 2)$ were $0.6 \%, 0.7 \%, 0.8 \%, 0.9 \%$ and $1.0 \%$, respectively, and the strain ratio was $R=-1$. The strain range is controlled by measuring the distance with the extensometer. For the tensile hold (TH) experiment, the loading stress was changed according to the value of the extensometer to ensure that the strain is kept constant.

The longitudinal metallographic sections were polished and chemically etched in a solution containing $5 \mathrm{~g} \mathrm{CuSO}_{4}$, $25 \mathrm{ml} \mathrm{HCl}, 20 \mathrm{ml} \mathrm{H}_{2} \mathrm{O}$ and $5 \mathrm{ml} \mathrm{H}_{2} \mathrm{SO}_{4}$, and the microstructural investigation was performed using a scanning electron microscope (SEM, JEOL JXA-8100). The fatigue fractured specimens were cleaned ultrasonically for 15-20 min in a mixture of acetone and ethanol, and they were observed using an FEI-nano 450 scanning electron microscope. Transmission electron microscopy (TEM) samples taken from transverse section of specimens, $3 \mathrm{~mm}$ far from fracture surface, by conventional grinding and twin-jet in a solution of $90 \% \mathrm{C}_{2} \mathrm{H}_{5} \mathrm{OH}+10 \% \mathrm{HClO}_{4}$ at a temperature of $-20{ }^{\circ} \mathrm{C}$ and a voltage of $20 \mathrm{~V}$, to investigate the deformation morphologies, including dislocations and stacking faults, were examined using a JEM-2100F TEM operating at a voltage of $200 \mathrm{kV}$. The electron diffraction patterns and energydispersive X-ray spectroscopy (EDS) of selected areas were obtained from the TEM samples to confirm the phase crystal structure and composition of the aged samples, respectively.

\section{Results}

\subsection{Microstructure Before Test}

Figure 1 shows the macrostructures and microstructures of nickel-based SC superalloys. A typical $\gamma-\mathrm{Ni}_{\mathrm{SS}} / \gamma^{\prime}-\mathrm{Ni}_{3} \mathrm{Al}$
Table 1 Chemical composition (wt \%) of the alloy

\begin{tabular}{cccccccccc}
\hline Cr & Co & W & Mo & Ta & Re & Al & Hf & B & C \\
\hline 4 & 8 & 7 & 2 & 7 & 3 & 6 & 0.2 & 0.1 & 0.1 \\
\hline
\end{tabular}




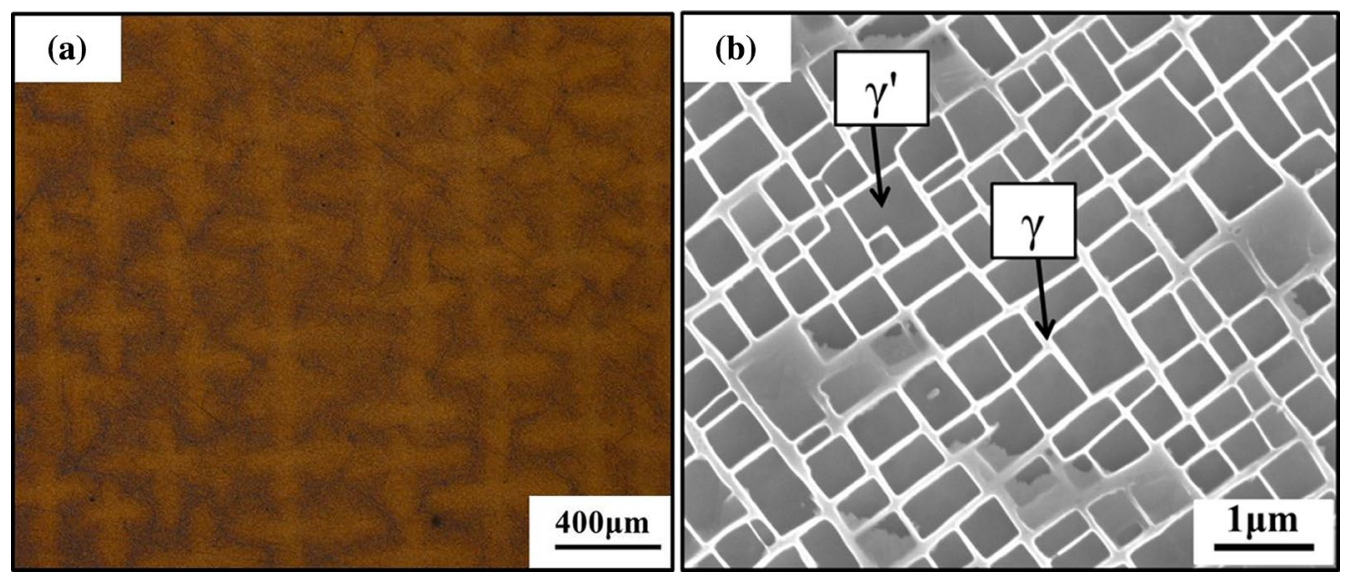

Fig. 1 Macrostructure a, microstructure b of nickel-based SC superalloy used in the experiment (the $\gamma$ and $\gamma^{\prime}$ phases were marked in (b))

two-phase microstructure is observed at the direction of [001], in which regular cuboidal $\gamma$ ' $-\mathrm{Ni}_{3} \mathrm{Al}$ precipitates uniformly align in the $\gamma-\mathrm{Ni}_{\mathrm{SS}}$ matrix. Statistical analysis confirms that $\gamma$ ' precipitates are approximately $350-500 \mathrm{~nm}$ in the cube edge length, and the $\gamma$ channel is $50-100 \mathrm{~nm}$ in width. The volume fraction of the $\gamma$ ' phase is approximately $70 \%$.

\subsection{Fatigue Behavior}

\subsubsection{Fatigue Life and Cyclic Stress Response Behavior}

Figure 2 presents various LCF curves of alloy at $760{ }^{\circ} \mathrm{C}$, comparing the LCF behaviors between both 60 -s TH and none tensile holding $(\mathrm{NTH})$ at the strain amplitude $(\Delta \varepsilon / 2)$ of $0.7 \%, 0.8 \%, 0.9 \%, 1.0 \%$ and $1.2 \%$. As shown in Fig. 2a, the LCF life of NTH experiment was higher than that of $60 \mathrm{~s} \mathrm{TH}$ experiment at any strain amplitude, which indicated a negative effect of holding at peak strain amplitude. Figure $2 b-f$ exhibits the cyclic stress response behaviors at
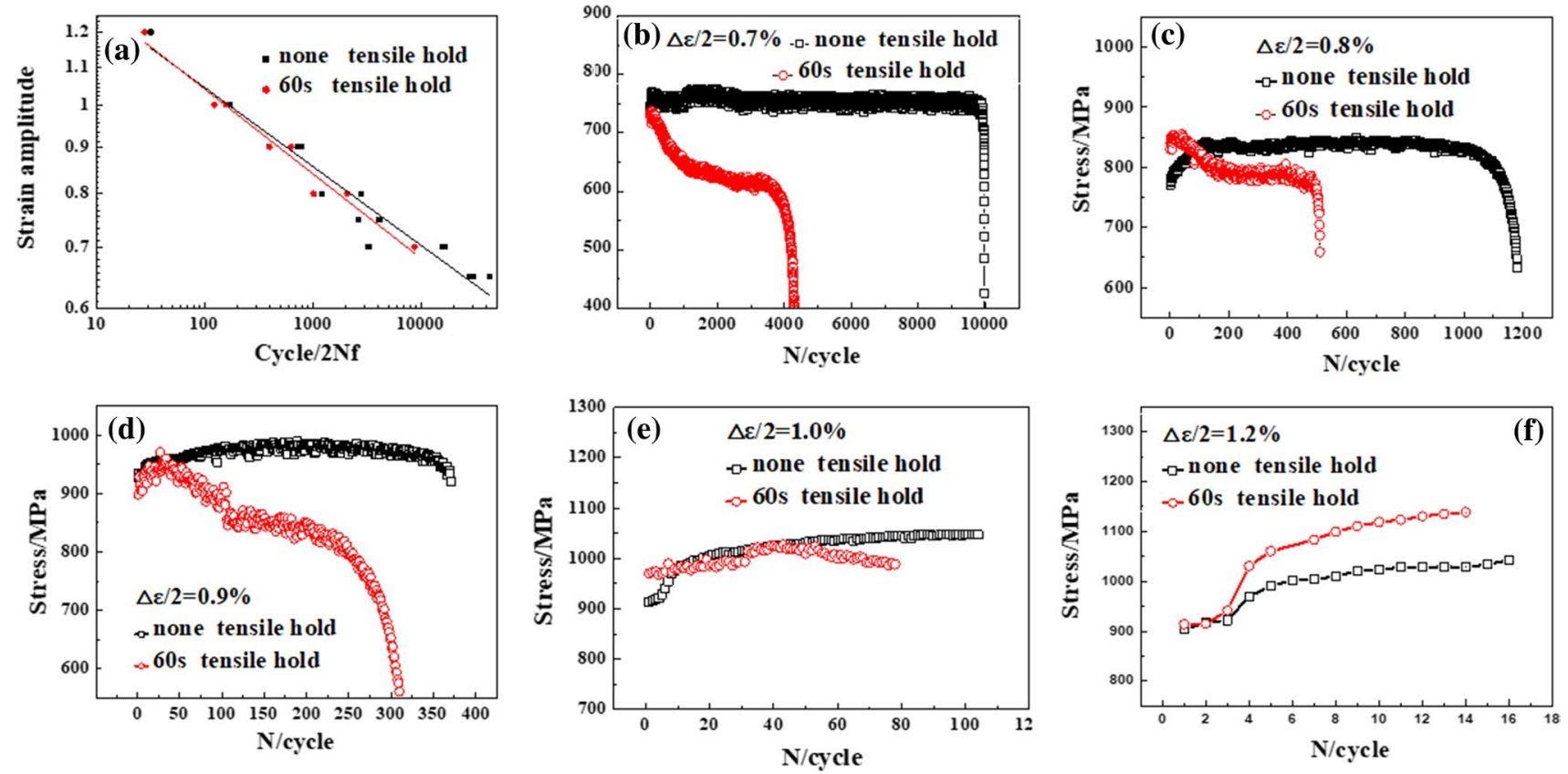

Fig. 2 a LCF life of the alloy at different strain amplitudes. b-f Cyclic stress response curves of the alloy at different amplitudes: $\mathbf{b} \Delta \varepsilon / 2=0.7 \%$, c $\Delta \varepsilon / 2=0.8 \%, \mathbf{d} \Delta \varepsilon / 2=0.9 \%$, e $\Delta \varepsilon / 2=1.0 \%, \mathbf{f} \Delta \varepsilon / 2=1.2 \%$ 
different strain amplitudes. It can be seen that the cyclic stress response was different as the strain amplitude increasing. For example, the cyclic stress response behavior of NTH experiment exhibited cyclic stable from 0.7 to $0.9 \%$. However, the cyclic softening behavior was exhibited at the strain amplitude of $0.7-0.9 \%$ with 60 -s TH alloy. At $\Delta \varepsilon / 2=1.0 \%$ and $1.2 \%$, both of NTH and $60-\mathrm{s}$ TH alloy exhibited the similar change and the cyclic hardening behaviors.

\subsection{Microstructure Evolution and Dislocation Structure}

To examine evolution of the $\gamma / \gamma^{\prime}$ coherent microstructure, fatigue tested specimens were sectioned longitudinally and observed. Figure 3 shows deformation morphologies at various strain amplitudes. Typical slip bands (SBs) were observed, and they were resulted from opposite sliding of the $\gamma / \gamma^{\prime}$ microstructure in the both sides of the slip band (See Fig. 3a, b and inset in Fig. 3c). The SBs might shear into the $\gamma$ ' phase and go along the $\gamma$ phase, as schematically illustrated in Fig. 3b. It has been confirmed that the SBs included plenty of dislocations caused by local stress concentration. Therefore, the SBs played a significant role in the plastic deformation and damage during fatigue [28].

Dislocation configurations in the gage section of the $\mathrm{NTH}$-fatigued specimens at the strain amplitudes of $0.7-1.2 \%$ were characterized (Fig. 4). As shown in Fig. 4, the mode of dislocation movement was different as the strain amplitudes changed. At the low strain amplitude of $0.7 \%$, the slip band (SB) was present after being projected on (001) plane along [110] direction, indicating that the planar slip was the main deformation mechanism in low strain amplitude level, as shown in Fig. 4a. This SB consisted plenty of dislocations restricted to one slip system, slipping in the matrix and cutting through the $\gamma^{\prime}$ phase. The SFs that were inside the $\gamma$ ' phase or $\gamma$ phases along the same direction were observed at the SB. At the same time, those dislocations of zigzag characteristics indicated that these dislocations were moving by cross-slip in the $\gamma$ channels. The threedimensional schematic illustrated the directions of dislocation movement were at different $<110>$ directions in $\{111\}$ planes (Fig. 4b).

Figure $4 \mathrm{c}$ and $\mathrm{d}$ shows typical dislocation structures as the strain amplitude was $0.9 \%$ after LCF tests. As shown in Fig. 4c, plenty of matrix dislocations were moving by crossslip in the $\gamma$ phase on different $<110>$ directions in $\{111\}$ planes (demonstrated by the schematic) and the interface dislocations were piled up at the interface of $\gamma / \gamma^{\prime}$ phase at the strain amplitude of $0.9 \%$ (Fig. 4d). The distribution of dislocation was homogeneous and the density of dislocation increased progressively comparing with the strain amplitude lower than $0.9 \%$. Little dislocation cut through $\gamma$ ' phase can also be observed as shown in Fig. 4h when the total strain amplitude was as high as $1.0 \%$. At the strain amplitude of $1.2 \%$, plenty of dislocations were piling up at the interface of $\gamma / \gamma^{\prime}$ phase and the $\gamma$ channel. It was difficult to move at the $\gamma$ channel thus little dislocation cut through the $\gamma$ ' phase.

Figure 5 shows the dislocation configuration of the fatigue rupture specimens with $60 \mathrm{~s} \mathrm{TH}$ at the strain amplitude from 0.7 to $1.2 \%$. Under the strain amplitude of $0.7 \%$, the cubic of $\gamma^{\prime}$ phase was not degraded and the width of $\gamma$ channel remained unchanged. The typical configuration of dislocation loop was refined in the $\gamma$ channel, and the density was low as shown in Fig. 5a. The glide of dislocations bowed out when they meet the $\gamma$ ' phase. In addition, there was also less SF in the $\gamma$ ' phase than NTH specimens as shown in Fig. 5b. Figure $5 \mathrm{c}$ shows the configuration of dislocation at $0.9 \%$ with $60 \mathrm{~s} \mathrm{TH}$ at $760{ }^{\circ} \mathrm{C}$, the SFs appeared in the $\gamma$ ' phase and some dislocation can also be observed to cut through the $\gamma$ ' phase. It was worth noting that the dislocation and the SF have the interaction with each other. It indicated that SFs does not hinder the movement of movable dislocation in $\gamma^{\prime}$ phase. In additional, at local area of surface of $\gamma^{\prime}$ phase and $\gamma$ matrix, there were plenty of dislocation loops and the
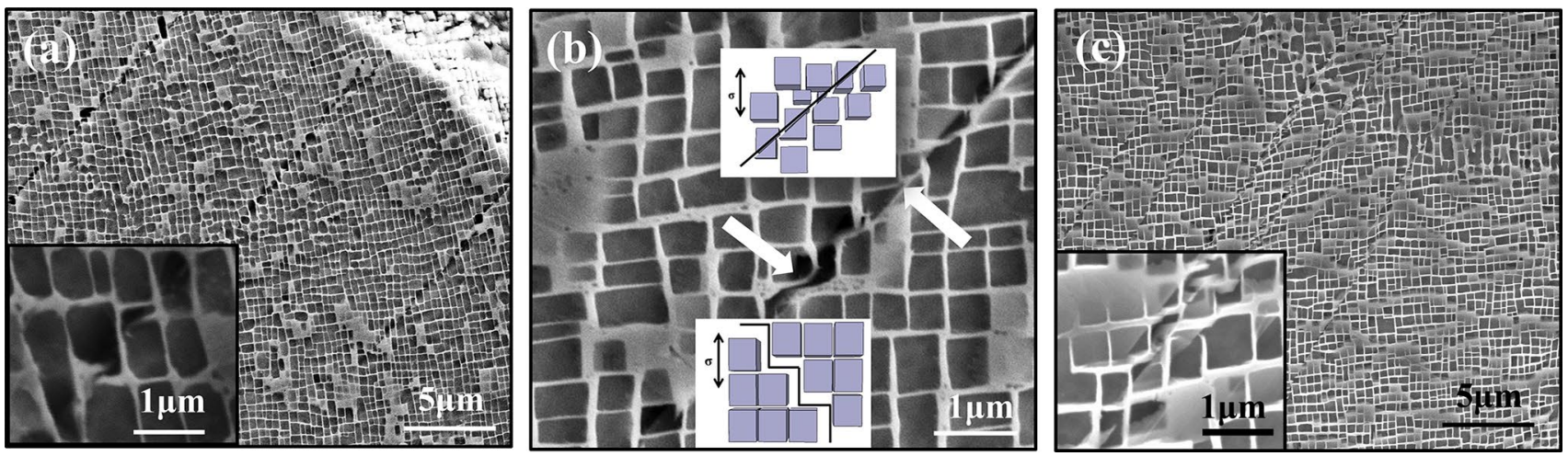

Fig. 3 Fracture characteristics of the longitudinal profile of the fatigue: a $\Delta \varepsilon / 2=0.7 \%, \mathrm{NTH}$, near the fracture surface, $\mathbf{b} \Delta \varepsilon / 2=0.8 \%$, NTH, ruptured by shearing $\gamma^{\prime}$ and along $\gamma, \mathbf{c} \Delta \varepsilon / 2=1.2 \%, 60 \mathrm{~s} \mathrm{TH}$, near the fracture surface 

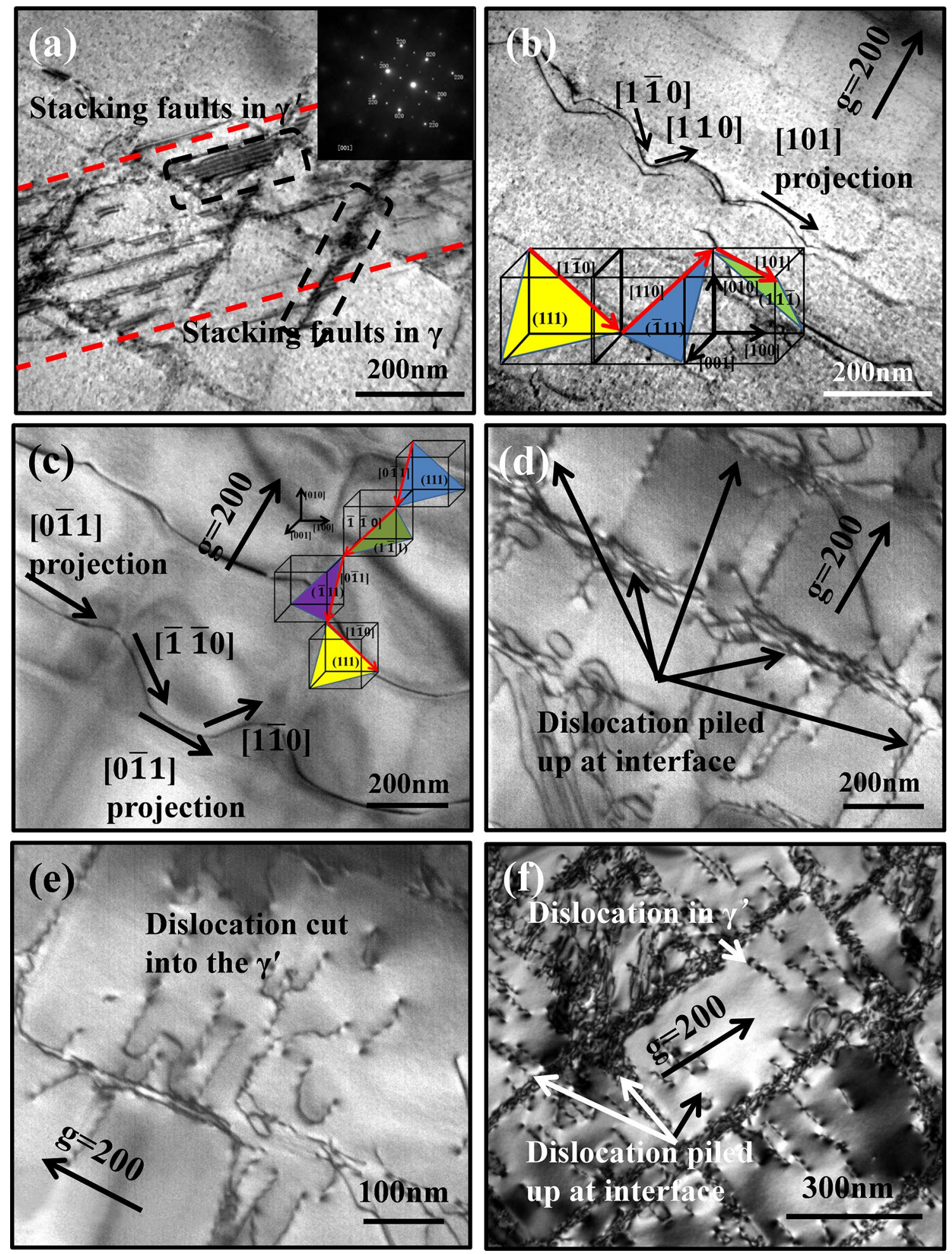

Fig. 4 Dislocation configurations of the experimental alloy after LCF failure at different strain amplitudes: a, b $\Delta \varepsilon / 2=0.7 \%$, NTH; c, d $\Delta \varepsilon / 2=0.9 \%, \mathrm{NTH} ; \mathbf{e} \Delta \varepsilon / 2=1.0 \%, \mathrm{NTH} ; \mathbf{f} \Delta \varepsilon / 2=1.2 \%$, NTH

irregular dislocation networks at the $\gamma$ channel and the dislocation density was larger than the other area as shown in Fig. 5d. SFs and APB dislocations cut $\gamma$ ' and the dislocation glide at on $\gamma$ channel on multiple $\{111\}<110>$ slip systems were also the main deformation modes. At the strain amplitude of $1.2 \%$, there were also some APB dislocations and 

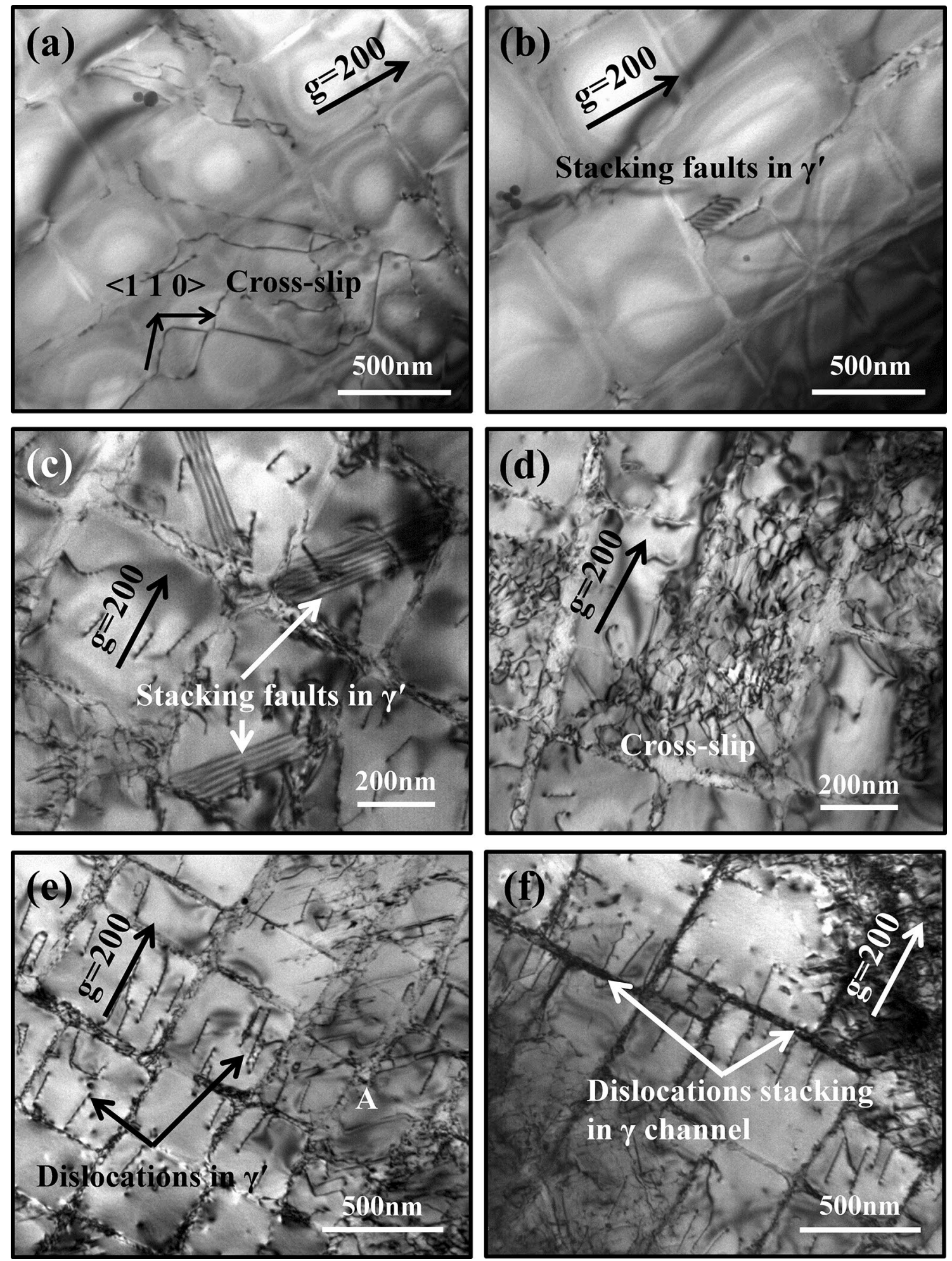

Fig. 5 Dislocation configurations of the experimental alloy after LCF failure at different strain amplitudes: a, b $\Delta \varepsilon / 2=0.7 \%, 60 \mathrm{~s}$ TH; c, d $\Delta \varepsilon / 2=0.9 \%, 60 \mathrm{~s} \mathrm{TH} ; \mathbf{e} \Delta \varepsilon / 2=1.0 \%, 60 \mathrm{~s} \mathrm{TH} ; \mathbf{f} \Delta \varepsilon / 2=1.2 \%, 60 \mathrm{~s} \mathrm{TH}$

SFs being observed to cut through the $\gamma$ ' phase (as shown in Fig. 5e, f). In additional, the channel of $\gamma$ and the cubic of $\gamma$ ' was unchanged and the stress of this condition increased; therefore, the dislocation bowing out at $\gamma$ channel on the $\{111\}$ plane when encountering the $\gamma$ ' precipitates. Plenty of dislocations were confined into the $\gamma^{\prime} / \gamma$ interface and moved 
hardly as the tensile and compression stress was high. At the $\gamma$ phase, there were lots of dislocation networks forming.

\subsection{Fatigue Fracture Morphology Under Different Strain Amplitudes}

The fracture morphologies of none tensile hold and with 60 -s tensile holding specimens are shown in Fig. 6a-f. The typical fatigue fracture surface included fatigue source, crack propagation region and instant rupture region, as marked in Fig. 6a, b, d and e. The flat fatigue source initiated on the $\{001\}$ planes of the specimen surface, as a result of repeated tension and compression loadings, as observed in Fig. 6a, $\mathrm{b}$, and more clear at Fig. $6 \mathrm{c}$. The morphologies of fatigue source at Fig. 6a demonstrated that the stress concentration area caused by the porosity. However, there were oxidation and porosity at the surface of a fatigue source region with 60-s tensile hold specimens as shown in Fig. 6f. Those areas took place plastic deformation and resulted in the crack initiation. The fatigue cracks propagated and fracture was around $45^{\circ}$ with the loaded stress axis and determined to be along $\{111\}$ planes (Fig. 6a, b, e). Furthermore, the slip stage along the different $\{111\}$ planes demonstrated that the number of active slip systems along $\{111\}$ increased. The total area of fatigue source and propagation region was found to reduce with increasing strain amplitude, it can be found that the fatigue source disappeared and the creep played an important role in the crack propagation process.

\section{Discussion}

\subsection{Effect of Strain Amplitude on Dislocation Movement}

On the basis of microstructural evolution, the deformation and damage behaviors of the alloy during LCF processing can be confirmed. The deformation mechanisms including planar slip and wavy slip were divided by mode of dislocation movement under different strain amplitudes. The dislocation movement changed gradually from the planar slip to the wavy slip with the strain amplitude increasing with none tensile hold time (Fig. 6). However, as for the hold time specimens, both the planar and the wavy slip can be observed at the deformation microstructures. The crystallographic slip plane plays an important role at the progress of fatigue crack propagation and the fatigue striation features can be observed at fracture surface. The influence of dislocation for cyclic response behavior was discussed in the following discussion.
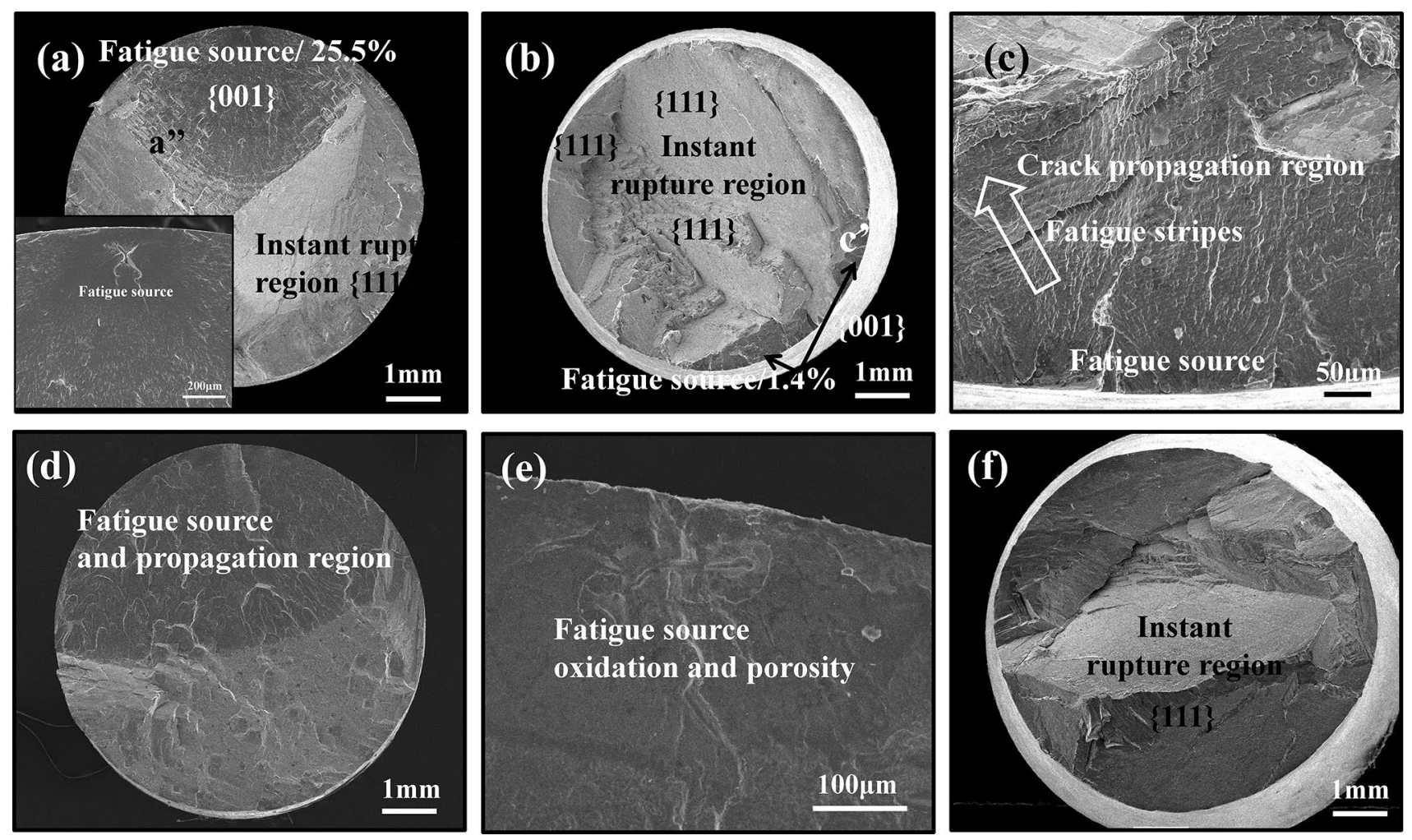

Fig. 6 Fatigue fracture morphologies under different load conditions: a $\Delta \varepsilon / 2=0.7 \%$, none tensile hold specimen; $\mathbf{b}$, $\mathbf{c} \Delta \varepsilon / 2=1.0 \%$, none tensile hold specimen; $\mathbf{d}$, e $\Delta \varepsilon / 2=0.7 \%, 60$ s tensile hold specimen; $\mathbf{f} \Delta \varepsilon / 2=1.2 \%, 60$-s tensile hold specimen 


\subsubsection{Cyclic Softening and Stable Behavior}

The annihilation and multiplication of dislocation leading to the transformation of cyclic stress response behaviors.

Under the strain amplitude of $0.7 \%$, the deformation mechanism of none tensile holding and the 60-s tensile holding included wavy and planar slip. However, in the process of deformation of NTH experiments, SFs will be larger and the cross-slip dislocation accompanied by annihilation as the number of cycles increasing, which played an important role at stable cyclic behavior.

As described in the previous section, the SFs bounded by partial dislocation were formed within these slip band and cut through $\gamma$ ' phase during LCF at the strain amplitude of $0.7 \%$, which suggested that the stacking fault energy decreased and the SFs formed in this alloy with additional Re and Co. On the other hand, at the process of cycle, there was a need for more stress due to the growth of SFs at the $\gamma$ ' phase. The formation mechanism of SFs at the $\gamma^{\prime}$ phase was as follows. Firstly, the a/2 <110> dislocation was hindered at the interface of the $\gamma^{\prime}$ and $\gamma$ phases and resolved into two partial dislocations; then one of the partial dislocations cut into the $\gamma$ ' phase and the SFs formed between partial dislocations. The SFs can also form due to the dislocation dissociation in $\gamma$ phase due to SFs energy decreased when the segregation of Re and Co in $\gamma$ phase [26].

Furthermore, the zigzag characteristic dislocations moved by cross-slip in the $\gamma$ channel, indicating that the movement of partial dislocation was active at different $\{111\}$ planes. The screw dislocations can relieve the misfit of $\gamma / \gamma$ ' interface partially [29]. It could be concluded that both of the SFs and the screw dislocation caused cyclic stable at low strain amplitude.

The annihilation and multiplication of dislocation led to the transformation of cyclic stress response behaviors. Under the strain amplitude of $0.7 \%$, the deformation mechanism of NTH and the 60-s TH included wavy and planar slip.

It is also indicated that the interaction of SFs and dislocation may cause cyclic softening. Under the strain amplitude of $0.9 \%$, the density of SFs was larger than the strain amplitude of $0.7 \%$ at $\gamma$ ' phase. The dislocation of APB has enough stress to cut through $\gamma$ ' phase and interacts with SFs. The SFs cannot hinder the movement of dislocation, indicating that the stress of LCF was low and the cyclic softening behavior occurred.

For the different tensile hold time specimens of $0.8 \%$ strain amplitude, the cyclic stress response behavior was softening as the cycle increased. At the condition of tensile hold of $0.8 \%$ strain amplitude, the SFs can cut through the $\gamma$ ' phase and $\gamma$ phase continue which indicated that the misfit of $\gamma^{\prime} / \gamma$ interface was decreased during the process of $\mathrm{TH}$ experiment.

\subsubsection{Cyclic Hardening Behavior}

When the strain amplitude was higher than $0.8 \%$ of none tensile hold LCF, there were three typical characteristics of the dislocation movement. The number of dislocations piling up around the cuboidal $\gamma^{\prime} / \gamma$ interface increased due to the higher strength of $\gamma^{\prime}$ phase at $760{ }^{\circ} \mathrm{C}$ [30]. Meanwhile, the maximum stress increased with increasing the strain amplitude, and little dislocation can cut through the $\gamma^{\prime}$ phase. However, the strength of $\gamma^{\prime}$ phase increased, and the movement of dislocation was difficult in $\gamma$ ' phase. As a result, a higher stress was required to continue the dislocation movement. In addition, plenty of dislocations glided in the $\{111\}$ plane in the matrix and the movement of dislocation can be influenced by each other with increasing the strain amplitude. In a word, the $\gamma$ ' phase displays a remarkable role in the movement of dislocation and the cyclic behavior was hardening.

The wavy slip can be observed under all of the strain amplitudes. The cross-slip in $\{111\}$ planes was wavy when projected on the $\{001\}$ plane illustrated in Fig. 7. Furthermore, the density of dislocation increased with the improving strain amplitude, which indicated that more dislocation can be active at the same time in the different $\{111\}$ planes.

The cyclic stress response behavior was related to the cubic of $\gamma$ ' phase at high temperature. At this work, the cubic of $\gamma$ ' phase has no degradation as the change of amplitude and hold time. In addition, the strength of $\gamma$ phase was lower than the $\gamma$ ' phase. Thus, the most important reason for cyclic hardening was the hinder effect of the harder of $\gamma$ ' phase. As a result, the dislocation was active easily at $\gamma$ phase as the stress of LCF increased.

\subsection{Effect of Strain Amplitude on Fracture Morphology}

In the process of crack propagation, the fatigue failure mode changed from a mode I (perpendicular to the loading direction) in fatigue source region to the $\{111\}$ octahedron slip mode in the propagation and instant region. The transformation scale of crack from fatigue source region to propagation region was shortened as the resolved shear stress was improving and the dislocation was activated on multi$\{111\}$ planes. The reason for such a transformation can be explained by the mechanism of environmental damage and the change of the resolved stress. At fatigue source region, the crack was small, and the crack driving force parameter of the principal normal stress was low. It was difficult to reach the threshold of the crack propagation. As a result, the crack propagation at fatigue source persisted for a long time and the oxygen absorbed fully ahead of the crack-tip [31]. When the length of crack increased, the failure mode was changed from mode I to the crystallographic fracture. 


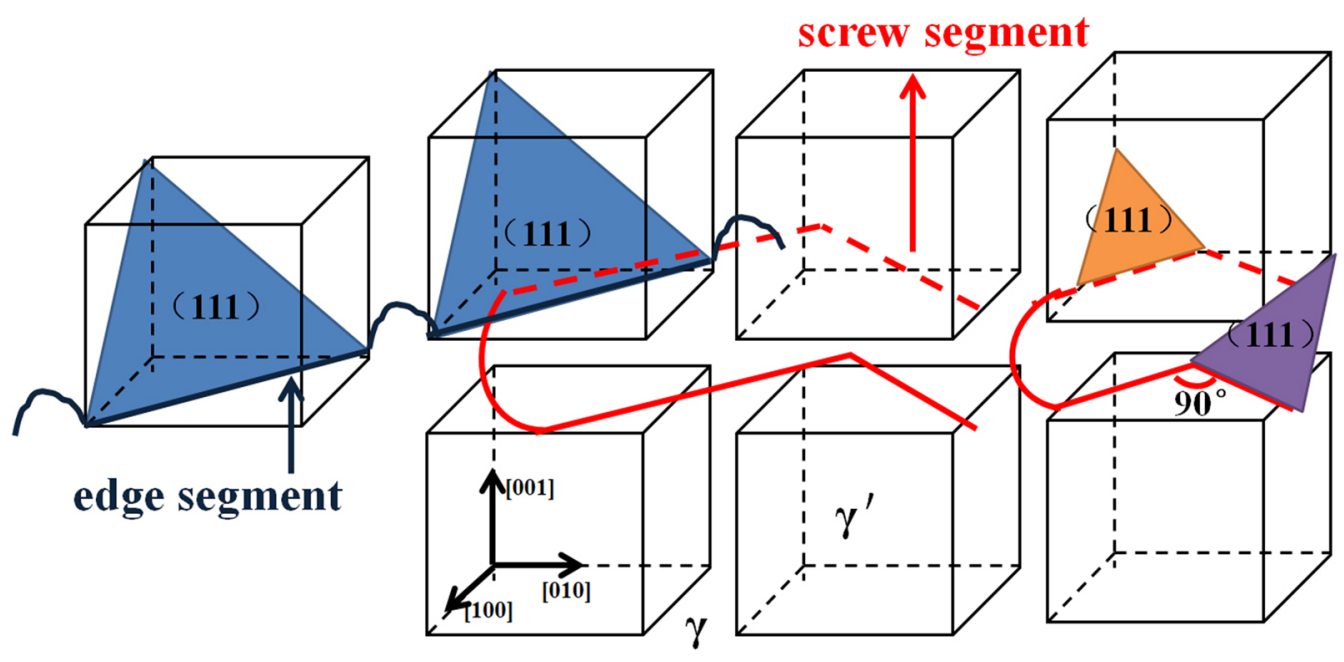

Fig. 7 Edge and screw dislocation movement in different $\{111\}$ at $760{ }^{\circ} \mathrm{C}$

It could be concluded that the parameter of resolved shear stress intensity reached the threshold at first compared to the normal stress and played an important role in propagation and instant region. In addition, the number of active slip systems along $\{111\}$ increased with the strain amplitude improving. The main movement mode of dislocation by cross-slip demonstrated that the dislocation was active at the multi- $\{111\}$ plane. As a result, the different planes can reach the threshold of the crack propagation at the same time.

The number of fatigue source increased with the increasing strain amplitude. Since there was no passivation of oxidation for the micro-porosity in this alloy, the stress concentration position on the surface and subsurface had plastic deformation during cycle loading. The fatigue source formed at the stress concentration and the crack began to propagate. With the strain amplitude improved, the value of stress reached the threshold of propagation at more than one stress concentration position, which explains the occurrence of more than one source with the improving strain amplitude.

\section{Conclusions}

The mechanisms of deformation and damage at high temperature of the nickel-based superalloys were investigated under different strain amplitudes at $760{ }^{\circ} \mathrm{C}$. The following conclusions can be drawn from the present study:

1. As the $\Delta \varepsilon / 2 \leq 1.0 \%$, the interaction of fatigue-creep reduced the fatigue life significantly. While the $\Delta \varepsilon / 2>1.0 \%$, the fatigue life of the fatigue-creep was the same as that under the condition of LCF.

2. The cyclic stress response behavior of NTH experiment exhibited cyclic stable from 0.7 to $0.9 \%$, and the cyclic softening behavior was exhibited with 60-s TH alloy. At $\Delta \varepsilon / 2=1.0 \%$ and $1.2 \%$, both of NTH and 60-s TH alloy exhibited cyclic hardening behavior.

3. As the strain amplitude increased, the deformation mechanisms changed gradually from the planar to wavy slip. When the strain amplitude was lower than $0.8 \%$, the movement of cross-slip and the hardening effect of SFs led to cyclic stability. When the strain amplitude was higher than $0.8 \%$, plenty of dislocations piling up at the interface was the main reason for cyclic hardening.

\section{References}

[1] L.K. Ning, Z. Zheng, T. Jin, S. Tang, E.Z. Liu, J. Tong, Y.S. Yu, X.F. Sun, Acta Metall. Sin. 50, 1011 (2014). (in Chinese)

[2] H.W. Zhang, X.Z. Qin, X.W. Li, L.Z. Zhou, Acta Metall. Sin. 53, 684 (2017). (in Chinese)

[3] H. Zhang, Q.Y. Xu, Z.X. Shi, B.C. Liu, Acta Metall. Sin. 50, 345 (2014). (in Chinese)

[4] J.M. Lee, S. Wee, J.H. Yun, H. Song, Y. Kim, J.M. Koo, C.S. Seok, Int. J. Precis Eng. Manuf. Green Technol. 5, 311 (2018)

[5] A.K. Vasudevan, K. Sadananda, N. Iyyer, Int. J. Fatigue 82, 120 (2016)

[6] P.A.S. Reed, Mater. Sci. Technol. 25, 258 (2013)

[7] K.O. Findley, J.L. Evans, A. Saxena, Int. Mater. Rev. 56, 49 (2013)

[8] M. Kaminski, P. Kanouté, S. Kruch, E.P. Busso, J.L. Chaboche, Mater. High Temp. 33, 412 (2016)

[9] P. Li, Q.Q. Li, T. Jin, Y.Z. Zhou, J.G. Li, X.F. Sun, Z.F. Zhang, Int. J. Fatigue 63, 137 (2014)

[10] E. Fleury, L. Rémy, Mater. Sci. Eng. A 67, 23 (1993)

[11] S. Steuer, P. Villechaise, T.M. Pollock, J. Cormier, Mater. Sci. Eng. A 645, 109 (2015)

[12] M.R. Joyce, X. Wu, P.A.S. Reed, Mater. Lett. 58, 99 (2004)

[13] P.A.S. Reed, M.D. Miller, in Comparison of Low Cycle (Notch) Fatigue Behaviour at Temperature in Single Crystal Turbine Blade 
Materials, Paper presented at 13th International Symposium on Superalloys, Pennsylvania, 11-15 September 2008

[14] S. Miiller, J. Rosler, C. Sommer, W. Hartnagel, The Influence of Load Ratio, Temperature, Orientation and Hold Time on Fatigue Crack Growth of CMSX-4, IMS (The Minerals, Metals and Materials Society), Superalloys 2000

[15] V. Brien, B. Décamps, Mater. Sci. Eng. A 316, 18 (2001)

[16] S. Yandt, X.J. Wu, N. Tsuno, A. Sato, Cyclic Dwell Fatigue Behaviour of Single Crystal Ni-Base Superalloys With/Without Rhenium Superalloys (Wiley, New York, 2012)

[17] M. Ott, H. Mughrabi, Mater. Sci. Eng. A 272, 24 (1999)

[18] J. Zhang, Z. Hu, Y. Xu, Z. Wang, Metall. Mater. Trans. A 23, 1253 (1992)

[19] D. Mukherji, H. Gabrisch, W. Chen, H.J. Fecht, R.P. Wahi, Acta Mater. 45, 3143 (1997)

[20] J. Telesman, L.J. Ghosn, J. Eng. Gas Turbines Power 118, 399 (1996)

[21] T. Goswami, H. Hanninen, Mater. Des. 22, 217 (2001)

[22] B.F. Antolovich, A. Saxena, S.D. Antolovich, J. Mater. Eng. Perform. 2, 489 (1993)
[23] V. Lupinc, G. Onofrio, Mater. Sci. Eng. A 202, 76 (1995)

[24] R. Ohtani, N. Tada, M. Shibata, S. Taniyama, Fatigue Fract. Eng. Mater. Struct. 24, 867 (2001)

[25] P. Zhang, G. Chen, H.Y. Qin, C.J. Wang, Mater. Trans. 57, 25 (2016)

[26] S. Ma, L. Carroll, T.M. Pollock, Acta Mater. 55, 5802 (2007)

[27] X.G. Wang, J.L. Liu, T. Jin, X.F. Sun, Y.Z. Zhou, Z.Q. Hu, J.H. Do, B.G. Choi, I.S. Kim, C.Y. Jo, Scr. Mater. 99, 57 (2015)

[28] D.W. Maclachlan, D.M. Knowles, Fatigue Fract. Eng. Mater. Struct. 24, 503 (2008)

[29] H. Zhou, H. Harada, Y. Ro, I. Okada, Mater. Sci. Eng. A 394, 161 (2005)

[30] P. Li, Q.Q. Li, T. Jin, Y.Z. Zhou, J.G. Li, X.F. Sun, Z.F. Zhang, Mater. Sci. Eng. A 603, 84 (2014)

[31] I.J. Telesman, T.P. Gabb, P. Bonacuse, J. Gayda, in Effect of Microstructure on Time Dependent Fatigue Crack Growth Behavior in a P/M Turbine Disk Alloy, Paper Presented at 13th International Symposium on Superalloys, Pennsylvania, 11-15 Sept 2008 